\title{
INEQUALITIES OF GAUSS-BONNET TYPE FOR A CONVEX DOMAIN
}

\author{
B. V. DEKSTER ${ }^{1}$
}

ABSTRACT. Let $N$ be a compact convex $n$-dimensional Riemannian manifold with a boundary $\partial N$ having normal curvatures $\geqslant \kappa>0$. Suppose the sectional curvature $>$ $-\kappa^{2}$ in $N$. Let $H$ be the integral mean curvature of $\partial N, V$ be the volume of $N, k_{\mathrm{sc}}$ be the scalar curvature and $\bar{k}_{R}(p), p \in N$, be the maximum Ricci curvature at $p$. Then

$$
H \geqslant \frac{n-2}{2} \kappa^{2} V-\frac{1}{2(n-1)} \int_{N} k_{\mathrm{sc}} d V, \quad H \geqslant(n-2) \kappa^{2} V-\frac{1}{n-1} \int_{N} \bar{k}_{R} d V .
$$

Let $N_{\text {- denote }} N$ with nonpositive sectional curvature. Let $G$ be the integral Gauss curvature of $\partial N_{-}$. Then $G \geqslant-\kappa^{n-2} \int_{N_{-}} \bar{k}_{R} d V$. These three estimates are sharp. For a ball in 3-dimensional hyperbolic space, the ratio of the right-hand part of each estimate to its left-hand part (i.e. $V\left(\kappa^{2}+3\right) / 2 H, V\left(\kappa^{2}+1\right) / H$ and $2 \kappa V / G$ respectively) approaches 1 as the radius $\rightarrow \infty$. The same ratios for the estimates

$$
H \geqslant-\frac{1}{2(n-1)} \int_{N} k_{\mathrm{sc}} d V \text { and } H \geqslant-\frac{1}{n-1} \int_{N} \bar{k}_{R} d V
$$

(rougher ones but without $\kappa$ ) approach $\frac{3}{4}$ and $\frac{1}{2}$ respectively.

\section{The results.}

1.1. All manifolds, their boundaries and submanifolds here are supposed to be of class $C^{\infty}$ unless otherwise stated. Mean, Gauss, Ricci and scalar curvatures are supposed to be defined as in $[5,3.6,3.7]$. Let $M$ be a connected compact $n$-dimensional, $n \geq 2$, manifold with a nonempty boundary $\partial M$. Let $\rho(\cdot, \cdot)$ be the distance in $M$. Put $M(t)=\{p \in M / \rho(p, \partial M) \geq t\}, 0 \leq t \leq r \stackrel{\text { def }}{=} \max _{q \in M} \rho(q, \partial M)$. Suppose that

(i) all $M(t)$ are locally convex;

(ii) normal curvatures of $\partial M(t), 0 \leq t<r$, at any of its regular points are not less than $\kappa \geq 0$.

Then $M$ is said to be convex throughout or $\kappa$-convex throughout.

1.2. One could prove that $M(t), t \in[0, r]$, at any of its boundary points, can be "supported by a sphere of curvature $\kappa$ ". However we will not use this property.

Notice that the conditions (i) and (ii) hold if the normal curvatures of $\partial M(0)=$ $\partial M$ are not less than $\kappa>0$ and the minimum sectional curvature in $M, k_{m}$ satisfies

$$
k_{m}>-\kappa^{2} \text {. }
$$

This kind of domain, named normal, was studied in [2, 3 and 4]. The condition (ii) holds for normal domains by Lemma 4 and the subsequent Remark in [2]. The condition (i) can be proved similarly to Lemma 9 in [1]. For that, one should apply Theorem 1(2) from [2] to the geodesic $X C$ constructed in [1].

Received by the editors February 25, 1981.

1980 Mathematics Subject Classification. Primary 53C40.

${ }^{1}$ Research supported by an NSF Grant. 
At the same time, the domain pasted of a semicircle of unit radius in hyperbolic plane of curvature -1 and a flat figure bounded by a segment of length 2 and the circumferences of radius 2 centered at its ends is $\frac{1}{2}$-convex throughout but not normal.

1.3. THEOREM. Let $m$ be an $n$-dimensional, $n \geq 2, \kappa$-convex throughout domain, $V$ be its volume, $\bar{k}_{R}(p)$ be the maximum Ricci curvature at $p \in M, H$ be the integral mean curvature of $\partial M$, and $G$ be its integral Gauss curvature. Then

$$
H \geq(n-2) \kappa^{2} V-\frac{1}{n-1} \int_{M} \bar{k}_{R} d V .
$$

Let $M_{-}$denote $M$ with nonpositive sectional curvature. Then

$$
G \geq-\kappa^{n-2} \int_{M_{-}} \bar{k}_{R} d V
$$

1.4. If the right-hand part of (1.3.1) or (1.3.2) is nonpositive then the estimate is trivial.

For the Euclidean case, (1.3.1) yields

$$
H \geq(n-2) \kappa^{2} V
$$

which is probably known. Notice however that a combination of the isoperimetric inequality with the fact that the area $S$ of $\partial M$ satisfies $H \geq \kappa S$ yields an estimate rougher than (1.4.1) for large $n$.

Let $M_{1}$ be the metric product of a locally Euclidean 2-dimensional torus and a segment. The equalities in (1.3.1) and (1.3.2) hold when $M=M_{-}=M_{1}$. Then all terms in (1.3.1) and (1.3.2) vanish. For domains homeomorphic to a ball, the estimates (1.3.1) and (1.3.2) are also sharp in the following sense. If $M=M_{-}$ is a ball in 3-dimensional hyperbolic space of curvature -1 then the ratio of the right-hand part of each estimate to its left-hand part (i.e. $\left(V+\kappa^{2} V\right) / H$ and $2 \kappa V / G$ respectively) goes to 1 as the radius of the ball goes to $\infty$. The same ratio for the rougher estimate

$$
H \geq-\frac{1}{n-1} \int_{M} \bar{k}_{R} d V
$$

goes to $\frac{1}{2}$.

1.5. Expressing Ricci curvature in terms of the scalar curvature $k_{s c}$ and sectional curvatures and combining it with (1.3.1) and (1.3.2) one can obtain more estimates. An example follows.

Denote by $v_{1}, v_{2}, \ldots, v_{n}$ an orthonormal set of vectors at a point $p \in M$ such that $v_{n}$ shows in the direction of the maximum Ricci curvature. Let $k_{i j}, 1 \leq i<$ $j \leq n$, be the sectional curvature in the plane of the vectors $v_{i}, v_{j}$ and let $\underline{k}$ be the minimum sectional curvature at $p$. Then

$$
k_{s c} / 2=\sum_{1 \leq i<j \leq n} k_{i j}=\bar{k}_{R}+\sum_{1 \leq i<j \leq n-1} k_{i j} \geq \bar{k}_{R}+(n-1)(n-2) \underline{k} / 2 .
$$

Combining it with (1.3.1) one has

$$
H \geq(n-2) \kappa^{2} V-\frac{1}{2(n-1)} \int_{M} k_{s c} d V+\frac{n-2}{2} \int_{M} \underline{k} d V .
$$


If $M$ is a normal domain $N$ then $\underline{k} \geq k_{m}>-\kappa^{2}$ (see (1.2.1)) and $\int_{N} \underline{k} d V \geq-\kappa^{2} V$. Then

$$
H \geq \frac{n-2}{2} \kappa^{2} V-\frac{1}{2(n-1)} \int_{N} k_{s c} d V
$$

and

$$
H \geq-\frac{1}{2(n-1)} \int_{N} k_{s c} d V
$$

If $N$ is a ball in 3-dimensional hyperbolic space of curvature -1 then the ratios of the right-hand parts in (1.5.1) and (1.5.2) to their left-hand parts go respectively to 1 and $\frac{3}{4}$ as the radius of the ball goes to $\infty$.

\section{Curvature of parallel surfaces.}

2.1. LEMMA. Let d be a compact (n-1)-dimensional manifold having a finite number of components. Its boundary $\partial d$ is supposed to be piecewise $C^{\infty}$ if nonempty. Put $D=d \times\left[t_{1}, t_{2}\right], 0 \leq t_{1}<t_{2}<r$, and let $f: D \rightarrow M$ be a $C^{\infty}$-diffeomorphism such that $S_{t} \stackrel{\text { def }}{=} f(d \times t) \subset \partial M(t)$ (see 1.1), $t \in\left[t_{1}, t_{2}\right]$, and $f_{*}(\partial / \partial t)$ is orthogonal to the hypersurface $S_{t}$. Denote by $H\left(S_{t}\right) \geq 0$ and $G\left(S_{t}\right) \geq 0$ the integral mean and the integral Gauss curvature of $S_{t}$. (They exist by compactness of $S_{t}$.) Then

$$
\begin{gathered}
\frac{d}{d t} H\left(S_{t}\right)=\frac{1}{n-1} \int_{S_{t}}\left[\sum_{i=1}^{n-1} k_{i}-2 \cdot \sum_{1 \leq i<j \leq n-1} \kappa_{i} \kappa_{j}\right] d S, \\
\frac{d}{d t} G\left(S_{t}\right)=\int_{S_{t}} \sum_{j=1}^{n-1} k_{j} \cdot \prod_{\substack{1 \leq i \leq n-1 \\
i \neq j}} \kappa_{i} d S,
\end{gathered}
$$

where $\kappa_{i} \geq 0$ is the normal curvature of $S_{t}$ in the ith principal direction and $k_{i}$ is the sectional curvature in the plane determined by that direction and $f_{*}(\partial / \partial t)$. (The product $\prod$ in (2.1.2) spreads over $i=1,2, \ldots, j-1, j+1, \ldots, n-1$. For $n=2$, this product is assumed to be equal to 1 and $\sum \kappa_{i} \kappa_{j}$ in (2.1.1) is assumed to be zero.)

2.2. Lemma 2.1 is not new. Variation of the $m$ th curvature of a hypersurface, $1 \leq m \leq n-1$, was investiagted in [6] but in a very general form which makes an explicit reference difficult. The following simple calculation seems more appropriate for our case of parallel surfaces.

2.3. Proof OF LeMMA 2.1. One may assume that $d \subset R^{n-1}$ because in the general case, one can split $d$ into a few pieces each of which is covered by a map of an atlas on $d$.

Let $y^{i}, i=1,2, \ldots, n$, be the coordinates in $f(D)$ borrowed from $R^{n}$ (with $y^{n} \in$ $\left.\left[t_{1}, t_{2}\right]\right)$. Put $Y_{1}=f_{*}\left(\partial / \partial y^{i}\right) \circ f^{-1}$ and $N=Y_{n}$. Obviously $|N|=1, N \perp Y_{i}, i=$ $1,2, n-1$, and $\nabla_{N} N=0$. Put $g_{i j}=\left\langle Y_{i}, Y_{j}\right\rangle, g=\operatorname{det}\left\|g_{i j}\right\|, c_{i j}=-\left\langle\nabla_{Y_{i}} N, Y_{j}\right\rangle$, $c=\operatorname{det}\left\|c_{i j}\right\|, i, j=1,2, \ldots, n-1$. Then

$$
\begin{aligned}
& G\left(S_{t}\right)=\int_{S_{t}} \frac{c}{g} d S=\int_{d} \frac{c}{\sqrt{g}} d y^{1} \cdots d y^{n-1} \\
& H\left(S_{t}\right)=\frac{1}{n-1} \int_{S_{t}} c_{i j} g^{i j} d S=\frac{1}{n-1} \int_{d} c_{i j} g^{i j} \sqrt{g} d y^{1} \cdots d y^{n-1},
\end{aligned}
$$




$$
\begin{gathered}
\frac{d}{d t} G\left(S_{t}\right)=\int_{d}\left(\frac{c^{\prime}}{g}-\frac{c g^{\prime}}{2 g^{2}}\right) \sqrt{g} d y^{1} \cdots d y^{n-1} \\
\frac{d}{d t} H\left(S_{t}\right)=\frac{1}{n-1} \int_{d}\left[c_{i j}^{\prime} g^{i j}+c_{i j} g^{i j^{\prime}}+c_{i j} g^{i j} \frac{g^{\prime}}{2 g}\right] \sqrt{g} d y^{1} \cdots d y^{n-1} .
\end{gathered}
$$

Take a point $p \in S_{t}$. Specialize the coordinates $y^{1}, \ldots, y^{n-1}$ so that $g_{i j}=g^{i j}=\delta_{j}^{i}$, $i, j=1,2, \ldots, n-1$, and the vectors $Y_{1}, Y_{2}, \ldots, Y_{n-1}$ show in the principal directions of $S_{t}$ at the point $p$. Then $c_{i j}=0$ for $i \neq j$ and $c_{i i}=\kappa_{i}$ at $p$.

$$
\begin{gathered}
c=\prod_{i=1}^{n-1} \kappa_{i}, \quad g=1, \quad g^{\prime}=\sum_{i=1}^{n-1} g_{i i}^{\prime} ; \\
c_{j j}^{\prime}=-N\left\langle\nabla_{Y_{j}} N, Y_{j}\right\rangle=-\left\langle\nabla_{N} \nabla_{Y_{j}} N, Y_{j}\right\rangle-\left\langle\nabla_{Y_{j}} N, \nabla_{N} Y_{j}\right\rangle \\
=-\left\langle R\left(N, Y_{j}\right) N, Y_{j}\right\rangle+\left\langle\kappa_{j} Y_{j}, \nabla_{N} Y_{j}\right\rangle
\end{gathered}
$$

$$
c_{j j}^{\prime}=k_{j}+\frac{1}{2} \kappa_{j} g_{j j}^{\prime}
$$$$
c^{\prime}=\sum_{j=1}^{n-1} c_{j j}^{\prime} \cdot \prod_{\substack{1 \leq i \leq n-1 \\ i \neq j}} \kappa_{i}=\sum_{j=1}^{n-1} k_{j} \cdot \prod_{\substack{1 \leq i \leq n-1 \\ i \neq j}} \kappa_{i}+\frac{1}{2} \sum_{j=1}^{n-1} \kappa_{j} g_{j j}^{\prime} \cdot \prod_{\substack{1 \leq i \leq n-1 \\ i \neq j}} \kappa_{i} ;
$$

$$
c^{\prime}=\sum_{j=1}^{n-1} k_{j} \cdot \prod_{\substack{1 \leq i \leq n-1 \\ i \neq j}} \kappa_{i}+\frac{1}{2} \sum_{j=1}^{n-1} g_{j j}^{\prime} \cdot \prod_{i=1}^{n-1} \kappa_{i} .
$$

(2.3.3) and (2.3.5) imply

$$
\frac{c^{\prime}}{g}-\frac{c g^{\prime}}{2 g^{2}}=\sum_{j=1}^{n-1} k_{j} \cdot \prod_{\substack{i \leq i \leq n-1 \\ i \neq j}} \kappa_{i}
$$

where the right-hand part does not depend on the choice of the coordinates $y^{1}, \ldots, y^{n-1}$. Therefore, together with (2.3.1), it yields (2.1.2)

$$
g_{i i}^{\prime}=N\left\langle Y_{i}, Y_{i}\right\rangle=2\left\langle\nabla_{N} Y_{i}, Y_{i}\right\rangle=-2 c_{i i}=-2 \kappa_{i} \text { at } p .
$$

Let $G_{i i}$ be the cofactor of $g_{i i}$ in $\operatorname{det}\left\|g_{i j}\right\|, i, j=1,2, \ldots, n-1$. Obviously $G_{i i}=1$ at $p$. One can check that $G_{i i}^{\prime}=g^{\prime}-g_{i i}^{\prime}$ at $p$. Then $g^{i i^{\prime}}=\left(G_{i i} / g\right)^{\prime}=-g_{i i}^{\prime}=2 \kappa_{i}$ at $p$. Together with (2.3.6), (2.3.3) and (2.3.4), it shows that the square bracket in (2.3.2) is equal to $\sum_{i=1}^{n-1} k_{i}-2 \sum_{1 \leq i<j \leq n-1} \kappa_{i} \kappa_{j}$ and do not depend on the choice of the coordinates $y^{1}, y^{2}, \ldots, y^{n-1}$. This implies (2.1.1).

\section{Proof of Theorem 1.3.}

3.1. Let $\Omega$ be the subset of the normal bundle of $\partial M$ such that $v \in \Omega$ if and only if the geodesic $\exp _{\pi(v)} t v, 0 \leq t \leq 1$, is a shortest path in $M$ between $\exp _{\pi(v)} v$ and $\partial M$. ( $\pi$ means the natural projection.) The following facts are known, see for example [1, §4].

(i) For any $p \in \partial M$, there exits the maximum number $\phi(p)>0$ such that $\Omega \supset v$ if $|v| \leq \phi(p), \pi(v)=p$ and $v$ shows inside $M$. 
(ii) The function $\phi(p)$ is continuous in $\partial M$ and

$$
0<\phi(p) \leq r=\max _{q \in M} \rho(q, \partial M) .
$$

(iii) $\Omega$ is compact, homeomorphic to $\partial M \times[0,1]$ and $\partial \Omega=\{v \in \Omega / v=0\} \cup \omega$ where $\omega=\{v \in \Omega /|v|=\phi(\pi(v))\}$.

(iv) The mapping $f \stackrel{\text { def }}{=} \exp _{\left.\partial M\right|_{\Omega \backslash \omega}}$ is a $C^{\infty}$-diffeomorphism.

(v) The set $C \stackrel{\text { def }}{=} \exp _{\partial M}(\omega)$ (called cut locus) is compact and has $n$-dimensional measure zero.

3.2. Fix some $\epsilon \in\left(0, \min _{p \in \partial M} \phi(p)\right)$. Due to the continuity of $\phi$, one can select a $C^{\infty}$-function $\phi_{\epsilon}: \partial M \rightarrow R$ such that $\phi-\epsilon<\phi_{\epsilon}<\phi$. Put $\Omega_{\epsilon}=\{v \in \Omega /|v| \leq$ $\left.\phi_{\epsilon}(\pi(v))\right\}$. By Sard's Theorem, the set of critical values of $\phi_{\epsilon}$ has linear measure zero. Therefore for any natural $m>2$ there exist numbers $0=t_{0}<t_{1}<\cdots<t_{m}=$ $\bar{\phi}_{\epsilon} \stackrel{\text { def }}{=} \max _{p \in \partial M} \phi_{\epsilon}(p)$ such that $t_{i}-t_{i-1}<2 \bar{\phi}_{\epsilon} / m, i=1,2, \ldots, m$, and each $t_{i}, i \neq m$, is a regular value of $\phi_{\epsilon}$. (One can first divide $\left[0, \bar{\phi}_{\epsilon}\right]$ into $m$ equal parts and then "vary a little" the points of division to get rid of the critical values.)

Put $d_{i}=\left\{p \in \partial M / \phi_{\epsilon}(p) \geq t_{i}\right\}, i=0,1, \ldots, m-1$. Obviously $d_{i}$ is nonempty, consists of a finite number of components and is a compact $(n-1)$-dimensional manifold with a $C^{\infty}$-boundary (if any).

3.3. Put $D_{i}=d_{i} \times\left[t_{i-1}, t_{i}\right]$ which can be identified with $D_{i}=\left\{v \in \Omega_{\epsilon} / \pi(v) \in\right.$ $\left.d_{i},|v| \in\left[t_{i-1}, t_{i}\right]\right\}, 1 \leq i \leq m-1$. Put $S_{t}^{i}=f\left(d_{i} \times t\right)=\left\{f(v) / \pi(v) \in d_{i},|v|=t\right\}$ where $f$ is as in 3.1 (iv), $1 \leq i \leq m-1, t \in\left[t_{i-1}, t_{i}\right]$. Obviously the hypersurface $S_{t}^{i} \subset \partial M(t)$ and $f_{*}(\partial / \partial t)$ is orthogonal to $S_{t}^{i}$. Denote by $H\left(S_{t}^{i}\right)$ and $G\left(S_{t}^{i}\right)$ the integral mean and the integral Gauss curvature of $S_{t}^{i}$ for the normal $f_{*}(\partial / \partial)$ (directed into $M(t)$ ). They exist by compactness of $S_{t}^{i}$ and are nonnegative by local convexity of $\partial M(t)$.

Lemma 2.1 is now applicable. Due to $\kappa$-convexity of $M$, the normal curvatures of $S_{t}^{i}$ are $\geq \kappa$. Obviously the sum $\sum_{i=1}^{n-1} k_{i}$ in (2.1.1) does not exceed $\bar{k}_{R}$. Then (2.1.1) yields

$$
\frac{d}{d t} H\left(S_{t}^{i}\right) \leq \frac{1}{\dot{n}-1} \int_{S_{t}^{i}}\left[\bar{k}_{R}-(n-2)(n-1) \kappa^{2}\right] d S .
$$

For $M=M_{-},(2.1 .2)$ yields

$$
\frac{d}{d t} G\left(S_{t}^{i}\right) \leq \kappa^{n-2} \int_{S_{t}^{i}} \bar{k}_{R} d S .
$$

3.4. Integrating (3.3.1) and (3.3.2) over $\left[t_{i-1}, t_{i}\right]$ and denoting volume by $V[\cdot]$, one has

$$
\begin{gathered}
H\left(S_{t_{i}}^{i}\right)-H\left(S_{t_{i-1}}^{i}\right) \leq \frac{1}{n-1} \int_{f\left(D_{i}\right)} \bar{k}_{R} d V-(n-2) \kappa^{2} V\left[f\left(D_{i}\right)\right], \\
\left.G\left(S_{t_{i}}^{i}\right)-G\left(S_{t_{i-1}}^{i}\right) \leq \kappa^{n-2} \int_{f\left(D_{i}\right)} \bar{k}_{R} d V \text { (for } M_{-}\right) .
\end{gathered}
$$

Obviously $S_{t_{i-1}}^{i} \subset S_{t_{i-1}}^{i-1}$ and therefore

$$
H\left(S_{t_{i-1}}^{i}\right) \leq H\left(S_{t_{i-1}}^{i-1}\right), \quad G\left(S_{t_{i-1}}^{i}\right) \leq G\left(S_{t_{i-1}}^{i-1}\right) .
$$

Neglecting $H\left(S_{t_{m-1}}^{m-1}\right) \geq 0$ and $G\left(S_{t_{m-1}}^{m-1}\right) \geq 0$, keeping in mind that $S_{0}^{1}=\partial M$ for sufficiently large $m$ and summing up each of (3.4.1) and (3.4.2) for all 
$i=1,2, \ldots, m-1$, one has now

$$
\begin{gathered}
-H \leq \frac{1}{n-1} \int_{P_{m}} \bar{k}_{R} d V-(n-2) \kappa^{2} V\left[P_{m}\right], \\
-G \leq \kappa^{n-2} \int_{P_{m}} \bar{k}_{R} d V \quad\left(\text { for } M_{-}\right)
\end{gathered}
$$

where the "pyramid" $P_{m}=\bigcup_{i=1}^{m-1} f\left(D_{i}\right)$.

3.5. Let us replace $m$ in turn by $m_{q}=2^{q} m, q=1,2, \ldots$. For each new $m_{q}$, the numbers $0=t_{0}, t_{1}, \ldots, t_{m_{q}}=\bar{\phi}_{\epsilon}$ are supposed to include all such numbers selected earlier. (One can place each new point $T$ of division in the center of each old segment $\left[t_{i-1}, t_{i}\right], i=1,2, \ldots, 2^{q-1} m$, and then "vary it a little" to avoid critical value but to comply with the condition that each of the segments $\left[t_{i-1}, T\right],\left[T, t_{i}\right]$ be shorter than $2 \bar{\phi}_{\epsilon} / 2^{q} m$. This is possible since $t_{i}-t_{i-1}<2 \bar{\phi}_{\epsilon} / 2^{q-1} m$.) Obviously

$$
P_{m_{1}} \subset P_{m_{2}} \subset \cdots \subset f\left(\Omega_{\epsilon}\right) \text {. }
$$

3.6. Let $p \in \operatorname{int} f\left(\Omega_{\epsilon}\right)$ and $v=f^{-1}(p)$. Then $|v|<\phi_{\epsilon}(\pi(v))$. Since $t_{i}-t_{i-1}<$ $2 \bar{\phi}_{\epsilon} / m_{q}$, there exists $i \in\left\{1,2, \ldots, m_{q}-1\right\}$ such that $t_{i-1} \leq|v| \leq t_{i}<\phi_{\epsilon}(\pi(v))$ for sufficiently large $m_{q}$. According to $3.2, \pi(v) \in d_{i}$ and by $3.3, v \in D_{i}$. Thus $p \in$ $f\left(D_{i}\right) \subset P_{m_{q}}$ and therefore $\bigcup_{q=1}^{\infty} P_{m_{q}} \supset$ int $f\left(\Omega_{\epsilon}\right)$. Together with (3.5.1), it implies that $P_{m}$ in (3.4.3) and (3.4.4) can be replaced by $f\left(\Omega_{\epsilon}\right)$.

3.7. Denote by $C_{\epsilon}$ the closed $\epsilon$-neighbourhood of the cut locus $C$. Obviously $f\left(\Omega_{\epsilon}\right) \cup C_{\epsilon}=M$. Since $\left|\bar{k}_{R}\right|<A=$ const in $M$,

$$
\left|\int_{M} \bar{k}_{R} d V-\int_{f\left(\Omega_{\epsilon}\right)} \bar{k}_{R} d V\right| \leq A V\left[C_{\epsilon}\right] .
$$

By 3.1(v), $V\left[C_{\epsilon}\right] \rightarrow 0$ as $\epsilon \rightarrow 0$. Therefore $f\left(\Omega_{\epsilon}\right)$ which appeared in (3.4.3) and (3.4.4) due to 3.6 can be replaced finally by $M$ and $M_{-}$respectively. That completes the proof.

\section{BIBLIOGRAPHY}

1. B. V. Dekster, An inequality of the isoperimetric type for a domain in a Riemannian space, Math. USSR-Sb. 19 (1973), 257-274.

2. Estimates of the length of a curve, J. Differential Geometry 12 (1977), 99-115.

3. The volume of a slightly curved submanifold in a convex region, Proc. Amer. Math. Soc. 68 (1978), 203-208.

4. - Upper estimates of the length of a curve in a Riemannian manifold with boundary, J. Differential Geometry 14 (1979), 149.-166.

5. D. Gromoll, W. Klingenberg and W. Meyer, Riemannische Geometrie im Grossen, Lecture Notes in Math., vol. 55, Spring-Verlag, Berlin and New York, 1968.

6. H. Rund, Invariant theory of variational problems on subspaces of a Riemannian manifold, Hamburger Mathematische Einzelschriften, Neue Folge, Heft 5, Vandenhoeck and Ruprecht, Göttingen, 1971.

Department of Mathematics, University of Notre Dame, Notre Dame, INDIANA 46556

Current address: Department of Mathematics, Erindale College of University of Toronto, Mississauga, Ontario, Canada L5L 1 C6 